\title{
Group identification with (incomplete) preferences
}

DOI:

10.1111/jpet.12387

\section{Document Version}

Accepted author manuscript

Link to publication record in Manchester Research Explorer

\section{Citation for published version (APA):}

Cho, W. J., \& Saporiti, A. (2019). Group identification with (incomplete) preferences. Journal of Public Economic Theory. https://doi.org/10.1111/jpet.12387

\section{Published in:}

Journal of Public Economic Theory

\section{Citing this paper}

Please note that where the full-text provided on Manchester Research Explorer is the Author Accepted Manuscript or Proof version this may differ from the final Published version. If citing, it is advised that you check and use the publisher's definitive version.

\section{General rights}

Copyright and moral rights for the publications made accessible in the Research Explorer are retained by the authors and/or other copyright owners and it is a condition of accessing publications that users recognise and abide by the legal requirements associated with these rights.

\section{Takedown policy}

If you believe that this document breaches copyright please refer to the University of Manchester's Takedown Procedures [http://man.ac.uk/04Y6Bo] or contact uml.scholarlycommunications@manchester.ac.uk providing relevant details, so we can investigate your claim.

\section{OPEN ACCESS}


Saporiti Alejandro ORCID iD: 0000-0002-9156-464X

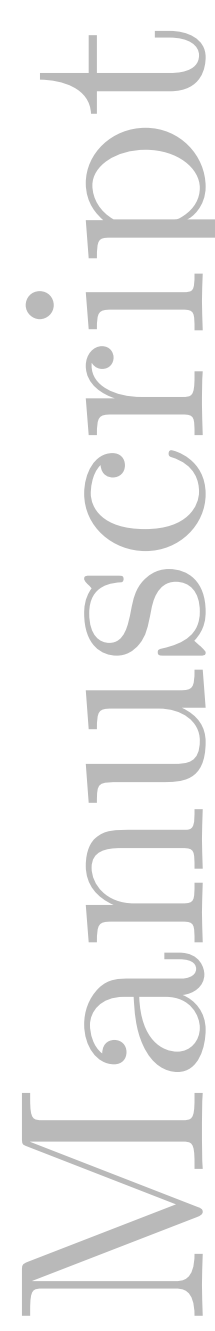

\author{
Wonki Jo Cho ${ }^{\dagger} \quad$ Alejandro Saporiti ${ }^{\ddagger}$
}

June 14, 2019

\begin{abstract}
We consider the problem of identifying members of a group based on individual opinions. Since agents do not have preferences in the model, properties of rules that concern preferences (e.g., strategy-proofness and efficiency) have not been studied in the literature. We fill this gap by working with a class of incomplete preferences derived directly from opinions. Our main result characterizes a new family of group identification rules, called voting-by-equitable-committees rules, using two well-known properties: strategy-proofness and equal treatment of equals. Our family contains as a special case the consent rules (Samet and Schmeidler, 2003), which are symmetric and embody various degrees of liberalism and democracy; and it also includes dictatorial and oligarchic rules that value agents' opinions differently. In the presence of strategy-proofness, efficiency turns out to be equivalent to non-degeneracy (i.e., any agent may potentially be included or excluded from the group). This implies that a rule satisfies strategy-proofness,
\end{abstract}

*We thank an Associate Editor and two anonymous referees whose comments greatly improved an earlier version of this paper. We also benefited from comments and suggestions by seminar participants at the University of Glasgow, the University of Liverpool, the 11th World Congress of the Econometric Society (Montreal), and the 2015 Economic Theory Workshop at the University of Manchester.

${ }^{\dagger}$ Department of Economics, Korea University, South Korea; chowonki@korea.ac.kr

${ }_{\ddagger}$ School of Social Sciences, University of Manchester, UK; alejandro.saporiti@manchester.ac.uk

This is the author manuscript accepted for publication and has undergone full peer review but has not been through the copyediting, typesetting, pagination and proofreading process, which may lead to differences between this version and the Version of Record. Please cite this article as doi: $10.1111 /$ jpet.12387

This article is protected by copyright. All rights reserved. 
efficiency, and equal treatment of equals if, and only if, it is a non-degenerate voting-by-equitable-committees rule.

JEL Classification Codes: C70, D70, D71.

Key Words: group identification; strategy-proofness; equal treatment of equals; symmetry; efficiency; voting-by-committees rules; consent rules.

\section{Introduction}

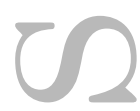

The axiomatic theory of group identification begins with the seminal article of Kasher and Rubinstein (1997), who relate the problem of ethnic and racial identity to social choice theory. ${ }^{1}$ The building blocks of this theory are (i) a group of agents, who seek to identify those with, or without, a certain qualification; (ii) the agents' opinions about each other, including themselves, which are the main input for qualifying or disqualifying an individual as a group member; and (iii) a rule that aggregates those opinions into a (social) decision. ${ }^{2}$ From the standpoint of an economist, an important disadvantage of this framework is that the lack of information about individual preferences prevents any analysis of incentive compatibility and efficiency of rules.

Motivated by this limitation, we derive from each agent's opinion a partial ordering that captures his preferences over decisions. Our preference specification ranks the agent's opinion as his most preferred decision; and it partially orders other decisions by looking at each agent's membership separately from the others' and by comparing it to the opinion. For instance, suppose that agent $i$ views agent $j$ as a member. Consider any pair of decisions that differ only in agent $j$ 's membership. Then agent $i$ prefers the decision that qualifies agent $j$ as a member to the decision that disqualifies him. ${ }^{3}$

With preferences defined, we proceed to study properties of rules that involve these preferences. The first one is an incentive property known as strategy-proofness. This property ensures that no agent ever benefit from misrepresenting his opinion. We find that strategy-proofness completely characterizes the family of voting-by-committees rules (Theorem 1). These rules appear first in the abstract social choice model of Barberà et

${ }^{1}$ See Dimitrov (2011) for a recent and comprehensive literature review and List (2008) for a discussion about the relationship between group identification and judgment aggregation.

${ }^{2}$ Opinions and decisions are represented by profiles of 0's and 1's, with 0 meaning "out" and 1 "in".

${ }^{3}$ This way of extending opinions to preferences resembles the way in which preference relations over alternatives are extended to preferences over preferences relations by Grandmont's (1978) notion of betweenness (Bossert and Sprumont, 2014) and preferences over alternatives are extended to preferences over lotteries by first-order stochastic dominance (Gibbard, 1977; Bogomolnaia and Moulin, 2001). Our discussion of the related literature provides further details on this matter.

This article is protected by copyright. All rights reserved. 
al. (1991), who study selecting a subset from a finite set of alternatives. In our setup, a voting-by-committees rule determines each agent's membership using a committee for the agent that consists of winning coalitions (subsets of agents). An agent is qualified as a member if he obtains the approval of a winning coalition in his committee.

Our proof for the characterization of the voting-by-committees rules hinges on two results that are interesting in their own right. First, strategy-proofness is equivalent to a local notion of incentive compatibility, called adjacent strategy-proofness (Proposition 1). Two opinions are adjacent if they differ in only one agent's membership. Adjacent strategy-proofness requires that no agent gain by reporting an opinion that is adjacent to the truth. In general, adjacent strategy-proofness is weaker than strategy-proofness. Yet on the universal domain, the two are equivalent. Moreover, this equivalence implies that a strategy-proof rule responds to changes in opinions in an intuitive way: when agent $i$ changes his opinion about agent $j$ while keeping his opinions about all other agents the same, agent $j$ 's membership, if it is affected, changes in the same direction while all the other agents' memberships remain unaffected. Conversely, any rule satisfying this property is strategy-proof (Corollary 1).

The second result upon which our characterization relies concern two properties proposed by Samet and Schmeidler (2003): monotonicity and independence. Monotonicity requires that a rule should adopt decisions that change (weakly) in the same direction as opinions do. Independence, on the other hand, requires that each agent's membership should be decided based solely on opinions about him. We show that strategy-proofness is equivalent to these two properties together (Proposition 2). As a by-product, this result provides a preference foundation for the two properties considered by Samet and Schmeidler (2003).

In addition to seeking to elicit individual opinions sincerely, we are also interested in rules that treat agents fairly. We consider two properties of fairness. The first property is equal treatment of equals. Two agents are equals if they are not distinguishable in any way, i.e., if they have the same opinion about all agents and all agents have the same opinion about them. ${ }^{4}$ Equal treatment of equals says that the membership decisions

${ }^{4}$ Kasher and Rubinstein (1997) call these two agents "symmetric". We use a slightly different terminology to avoid confusion with the concept of symmetry defined by Samet and Schmeidler (2003), which applies to rules, is stronger than equal treatment of equals, and is equivalent to the conjunction of essential anonymity and essential neutrality (Çengelci and Sanver, 2010). For voting-by-committees rules, essential anonymity requires that for each agent $i$, (i) a coalition containing $i$ belongs to the committee deciding $i$ 's membership if, and only if, any coalition of the same size containing $i$ belongs to that committee; and (ii) a coalition not containing $i$ belongs to the committee deciding $i$ 's membership if, and only if, any coalition of the same size not containing $i$ belongs to that committee. Essential neutrality requires the following: (i) for all agents $i$ and $j$, a coalition containing neither $i$ nor $j$ This article is protected by copyright. All rights reserved. 
for two equal agents should be the same. This axiom is not well-defined in the abstract model of Barberà et al. (1991) but it is meaningful in our model because of the additional structure that alternatives have in group identification. It also constitutes the most basic fairness requirement in our model.

We show that a rule satisfies strategy-proofness and equal treatment of equals if, and only if, it is a voting-by-committees rule whose associated committees satisfy the following equitable condition: for each pair of agents $i$ and $j$ and for each winning coalition $M$ that is in agent $i$ 's committee but not in agent $j$ 's, exactly one of the two agents belongs to $M$, so that whenever the two agents are equals, $M$ plays no role in determining their qualification (Theorem 2). These rules are new in the literature and we call them the voting-by-equitable-committees rules.

The family of consent rules proposed by Samet and Schmeidler (2003) is a special case in the class of voting-by-equitable-committees rules. Depending on the choice of the parameters, the consent rules can embody varying degrees of democracy and liberalism, accommodating different levels of social intervention in an agent's status. We characterize these rules by strategy-proofness and symmetry, a fairness notion stronger than equal treatment of equals (Theorem 3). Symmetry (Samet and Schmeidler, 2003) essentially requires that the names of the agents should not matter ("essentially" because an agent's opinion about himself plays a special role in the committee that deals with his own qualification). Our characterization of the consent rules relies on a characterization by Samet and Schmeidler (2003) and on our result that strategy-proofness is equivalent to monotonicity and independence combined.

Finally, we also investigate implications of efficiency. Because the preferences derived from opinions are incomplete, efficiency turns out to be a weak property. In fact, we show that in the presence of strategy-proofness, efficiency is equivalent to non-degeneracy (Proposition 4). Non-degeneracy requires that the membership decision should not be fixed for any agent. Therefore, using our previous results, we conclude that a rule satisfies strategy-proofness, efficiency, and equal treatment of equals if, and only if, it is a non-degenerate voting-by-equitable-committees rule (Theorem 4).

To summarize, our analysis in this work unveils a class of group identification rules that, in reference to Samet and Schmeidler (2003), goes "beyond liberalism and democracy". This family includes rules that are symmetric and non-symmetric; but all of them

belongs to the committee deciding $i$ 's membership if, and only if, it belongs to the committee deciding $j$ 's membership; and (ii) for all agents $i$ and $j$, a coalition belongs to the committee deciding $i$ 's membership if, and only if, there is a coalition of the same size that belongs to the committee deciding $j$ 's membership.

This article is protected by copyright. All rights reserved. 
satisfy strategy-proofness, efficiency, and equal treatment of equals. The subfamily of consent rules are symmetric voting-by-committees rules, for which only the size of winning coalitions (i.e., the quotas) matters, but not the names of the agents, except for the agent whose membership a committee deals with. By contrast, the voting-by-equitablecommittees rules allow committees to be oligarchic (e.g., the United Nations Security Council) or even dictatorial. These rules may be relevant when agents are heterogenous not only in their opinions, but also in other dimensions (e.g., power and expertise), which may justify weighting their opinions differently.

\section{Related Literature}

The first model of group identification is due to Kasher and Rubinstein (1997), who relate the problem of collective identity to social choice theory. The paper shows that when the range of a rule is restricted to be a proper subset of the set of agents, it satisfies independence and consensus (efficiency in our model) if and only if it is dictatorial. This result is known in the literature as the "who is a J" impossibility theorem. Saporiti (2012) enhances this result by weakening consensus and provides a proof that exploits the structure of decisive coalitions. Kasher and Rubinstein (1997) also propose equal treatment of equals (they call this property symmetry), a fairness requirement that a rule should not discriminate between two agents on any basis other than that represented by

opinions. Finally, they introduce and characterize the liberal rule, according to which an agent is a member if and only if he believes himself to be.

Samet and Schmeidler (2003) propose and characterize the family of consent rules, which contains the liberal rule as a special case. The consent rules are parameterized by the weights given to the individuals vis-à-vis the group for determining their membership, representing various levels of liberalism. Samet and Schmeidler (2003) point out the connection between the group identification model and Barberà et al. (1991). In particular, they discuss the possibility of misrepresenting opinions, but they do not characterize strategy-proof rules. In that sense, our work is an extension of their study. Also, Theorem 3 provides an alternative justification for the consent rules by showing that they are the only strategy-proof and symmetric rules.

In the papers cited above, there is a single group whose membership is to be decided. By contrast, Miller (2008) considers the setup where the group under question can vary. He imposes axioms that require decisions to be consistent with respect to conjunction and disjunction of groups. Cho and Ju (2017) study a model where multiple groups are identified simultaneously. They investigate consequences of an independence axiom, This article is protected by copyright. All rights reserved. 
similar to Arrow's (1951) independence of irrelevant alternatives, requiring that identification of each group should depend only on opinions about that group. Sung and Dimitrov (2005), Houy (2007), Çengelci and Sanver (2010), and Ju (2013), Cho and Ju (2015), and Cho and Park (2018) provide further characterizations and extenstions. See Dimitrov (2011) for a comprehensive review of the literature.

Our approach to deriving preferences over decisions from opinions resembles an avenue pursued in the study of non-manipulable social welfare functions. In a classical Arrovian framework with finitely many alternatives, a social welfare function associates with each profile of preference relations over alternatives a social preference relation. An agent's preference relation is defined only over alternatives. Therefore, outcomes of his manipulations, which are preference relations over alternatives, cannot be evaluated properly unless one makes an assumption on his preferences over preference relations over alternatives. Bossert and Storcken (1992) proceed by assuming that an agent prefers a preference relation to another if the former is closer to the true preference relation than the latter in the Kemeny distance sense. ${ }^{5}$ Athanasoglou (2016) adopts the same approach.

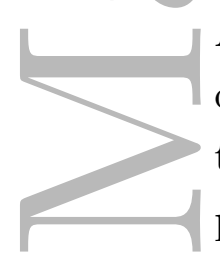

Another practice for evaluating preference relations over alternatives builds on the notion of betweenness due to Grandmont (1978). A preference relation is said to be between two preference relations if whenever the latter two preference relations agree on the preferences over a pair of alternatives, the former preference relation have the same preferences. With this idea in mind, one can say that an agent prefers a preference relation to another if the former lies between the latter and his true preference relation (so that it is closer to the truth) (Bossert and Sprumont, 2014). Unlike the preference notion based on the Kemeny distance, however, the preference notion based on betweenness gives rise to a partial relation over preference relations. Yet it is the most one can say unambiguously about an agent's preferences over preference relations. ${ }^{6}$

Once preferences over preference relations are defined, incentive and efficiency properties can be formulated. Take strategy-proofness for instance. For a complete preference notion, such as the one based on the Kemeny distance, the statement of strategy-proofness is standard and the individual and group versions of the property are studied by Bossert and Storcken (1992) and Athanasoglou (2016). For a partial preference notion, such as

${ }^{5}$ Given two preference relations over alternatives, the Kemeny distance between them is defined as the number of pairs of alternatives over which the two preference relations exhibit different preferences (Kemeny, 1959; Kemeny and Snell, 1962).

${ }^{6}$ For this reason, Bossert and Sprumont (2014) refer to the betweenness-based preference notion as the prudent extension.

This article is protected by copyright. All rights reserved. 
the one based on betweenness, two formulations are possibles: (i) no agent should ever gain by misrepresenting his preferences over alternatives; and (ii) each agent weakly prefers the outcome obtained by telling the truth to the outcome obtained by lying. Clearly, (ii) is a stronger requirement than (i) because it imposes comparability of any pair of outcomes. Bossert and Sprumont (2014) study social welfare functions satisfying (i). ${ }^{7}$

Our preference extension is similar to the procedure adopted by Bossert and Sprumont (2014) in that we construct, on the basis of opinions, a partial preference relation over decisions that contain only the unambiguous information under the separability assumption. Yet we differ from Bossert and Sprumont (2014) in the formulation of strategyproofness. We require a strong incentive property in line with formulation (ii) above, in order to ensure that no agent ever gain by manipulating a rule, irrespective of his preferences.

In a model where agents vote on subsets of abstract alternatives, Barberà et al. (1991) characterize the voting-by-committees rules on the domain of separable preferences by an incentive property and a full range condition (voter sovereignty). Since agents submit their preferences over all alternatives, their incentive property is weaker than our notion of strategy-proofness. Theorem 1 in our paper shows that the same family of rules is characterized by strategy-proofness alone when individual preferences are separable but represented by partial orders.

Introducing indifferences to the model of Barberà et al. (1991), Ju (2003) generalizes the concept of committee and characterizes the similarly-defined family of voting-bycommittees rules by strategy-proofness and an independence axiom. In Barberà et al. (1991), a fixed set of objects, over which agents' preferences are defined, serves as an agenda. Ju (2005) study consequences of varying the agenda. Associated to each agenda (a finite set of objects) is a profile of committees à la Barberà et al. (1991) that decides which objects in the agenda are chosen. The associated profile of committees may differ from one agenda to another and should have a special structure to satisfy resource monotonicity, a key axiom in $\mathrm{Ju}$ (2005) requiring that all agents be affected in the same

${ }^{7}$ Bossert and Storcken (1992) provides an impossibility result involving group strategy-proofness based on the Kemeny distance. Bossert and Sprumont (2014) characterize three families of social welfare functions by strategy-proofness based on betweenness and some other properties. However, as shown by Athanasoglou (2016), none of the three families satisfies the stronger notion of strategy-proofness based on the Kemeny distance when there are at least four alternatives. On the other hand, Sato (2015) considers several notions of strategy-proofness, including those in Bossert and Storcken (1992) and Bossert and Sprumon (2014), and show that they are equivalent under a "continuity" condition called bounded response (which requires that if individual preferences change by the smallest amount, so should the social preferences, if any).

This article is protected by copyright. All rights reserved. 
direction as the agenda expands.

A major distinction between these papers and ours, apart from the incomplete nature of our preference specification, is that we consider fairness properties - namely, equal treatment of equals and symmetry - and characterize the structures of committees emerging from them. We may extend our model in the spirit of $\mathrm{Ju}(2003,2005)$, allowing opinions to be one of "positive", "negative", and "neutral", or allowing the set of agents to vary. While we conjecture that the extension will preserve the spirit of many of our results, capturing the principle behind resource monotonicity in a group identification model will not be straightforward. ${ }^{8}$

Finally, Sato (2013), Carroll (2012), and Cho (2016) study the question of when a local incentive property is sufficient for a global incentive property. In particular, Sato (2013) identifies a condition on the preference domain that ensures the sufficiency for deterministic social choice rules, and Cho (2016) extends it to probabilistic rules. Our result that strategy-proofness and adjacent strategy-proofness are equivalent is related to these papers.

\section{The Model}

Let $N \equiv\{1, \ldots, n\}(n \geq 2)$ be a finite set of agents. There is a group that these agents seek to identify among themselves based on their opinions about group membership. To be more precise, each agent $i \in N$ has an opinion $\boldsymbol{p}_{\boldsymbol{i}} \equiv\left(p_{i j}\right)_{j \in N} \in\{0,1\}^{N}$, where for each $j \in N, p_{i j}=1$ (and $p_{i j}=0$ ) if agent $i$ believes (and does not believe, respectively) that agent $j$ belongs to the group. Let $\mathcal{D} \equiv\{0,1\}^{N}$ denote the domain of all opinions. An (identification) problem is a profile of opinions $\boldsymbol{p} \equiv\left(p_{i}\right)_{i \in N} \in \mathcal{D}^{N}$, which is treated as an $n \times n$ matrix, with row $i$ representing $p_{i}$. For each $i \in N$, denote by $\boldsymbol{p}^{i}$ column $i$ of $p$, which represents the opinions about agent $i$. A (social) decision is a profile $x \equiv\left(x_{i}\right)_{i \in N} \in\{0,1\}^{N}$, where for each $i \in N, x_{i}=1$ (and $x_{i}=0$ ) if agent $i$ is socially approved as a member (and a non-member, respectively) of the group. Let $\boldsymbol{X} \equiv\{0,1\}^{N}$ be the set of all decisions. An (identification) rule $\varphi: \mathcal{D}^{N} \rightarrow X$ associates with each problem $p \in \mathcal{D}^{N}$ a decision $\varphi(p) \equiv\left(\varphi_{i}(p)\right)_{i \in N} \in X$.

Below we use the following notation. For each $p \in \mathcal{D}^{N}$ and each $i \in N$, let $\boldsymbol{N}_{\mathbf{0}}(\boldsymbol{p}, \boldsymbol{i}) \equiv$ $\left\{j \in N: p_{j i}=0\right\}$ be the set of agents who believe that agent $i$ is not a member.

${ }^{8}$ Unlike in Barberà et al. (1991) or extensions thereof, agents in group identification are both voters with opinions and alternatives to be voted on. Thus, an expansion in the set of alternatives is always accompanied by an expansion of the same magnitude in the set of voters. This makes it unclear how a change in the population should affect membership decisions.

This article is protected by copyright. All rights reserved. 
Similarly, let $\boldsymbol{N}_{\mathbf{1}}(\boldsymbol{p}, \boldsymbol{i}) \equiv\left\{j \in N: p_{j i}=1\right\}$ be the set of agents who believe that agent $i$ is a member. For each $p \in \mathcal{D}^{N}$, each $i \in N$, and each $p_{i}^{\prime} \in \mathcal{D}$, let $\left(\boldsymbol{p}_{i}^{\prime}, \boldsymbol{p}_{-i}\right)$ be the problem where agent $i$ has opinion $p_{i}^{\prime}$ and for each $j \in N \backslash\{i\}$, agent $j$ has opinion $p_{j}$. Given $M \subseteq N$, let $\boldsymbol{p}_{M} \equiv\left(p_{i}\right)_{i \in M} \in \mathcal{D}^{M}$ be an opinion profile for the agents in $M$. Then $\left(\boldsymbol{p}_{M}^{\prime}, \boldsymbol{p}_{N \backslash M}\right) \in \mathcal{D}^{N}$ is the problem where each agent $i \in M$ has an opinion $p_{i}^{\prime}$ and each agent $j \in N \backslash M$ has an opinion $p_{j}$. The problem (and the decision) where all of its entries are 1 is denoted by $\mathbf{1}_{\boldsymbol{n} \times \boldsymbol{n}} \in \mathcal{D}^{N}$ (and $\mathbf{1}_{\mathbf{1 \times n}} \in X$, respectively). The problem $0_{n \times n}$ and the decision $0_{1 \times n}$ are similarly defined.

\section{Separability}

The model described so far lacks information on agents' preferences over decisions. This implies that properties of rules (such as strategy-proofness and efficiency) pertaining to these preferences cannot be studied. Yet there is a way to interpret opinions as preferences. Consider agent $i$ 's opinion $p_{i}$. It is natural to interpret $p_{i}$ as agent $i$ 's most preferred alternative in $X$. We do not know how he ranks the other alternatives in $X$. But if we place a restriction on admissible preferences, a "partial" preference relation can be recovered from $p_{i}$. The restriction we consider is separability: each agent's membership decision is valued independently of the other agents' membership decision. Formally, we assume that given $p_{i} \in \mathcal{D}$, for each $j \in N$ and each pair $x, y \in X$ such that $x_{j} \neq y_{j}$ and $x_{k}=y_{k}$ for each $k \in N \backslash\{j\}$, agent $i$ with opinion $p_{i}$ prefers $x$ to $y$ if, and only if, $x_{j}=p_{i j} \neq y_{j}$. Each separable preference relation that top-ranks $p_{i}$ is consistent with the strict preference relation $P_{i}$ defined as follows: for each pair $x, y \in X$,

$$
x P_{i} y \Longleftrightarrow \text { for each } j \in N, p_{i j} \neq x_{j} \text { implies } p_{i j} \neq y_{j} .
$$

We treat $P_{i}$ as the preferences over $X$ of agent $i$ with opinion $p_{i}$ and call it the preference extension of $\boldsymbol{p}_{\boldsymbol{i}}$. Denote the preference extensions of $p_{i}^{\prime}$ and $\hat{p}_{i}$ by $P_{i}^{\prime}$ and $\hat{P}_{i}$, and so on. Notice that $P_{i}$ is irreflexive, transitive, and incomplete. For example, suppose that $N=\{1,2\}$. Then according to the preference extension $P_{i}$ of opinion $p_{i}=(0,1)$, decision $(0,1)$ is most preferred, $(1,0)$ is least preferred, and $(0,0)$ and $(1,1)$ are not comparable. Our construction of the preference extension is related to the well-established notion of betweenness (Grandmont, 1978). Let $\succ_{i}$ be a strict preference relation over $X{ }^{9}$ For each $j \in N$, let $\boldsymbol{e}^{j} \in X$ be a decision whose entries are all zero except $e_{j}^{j}=1$. Then a strict preference relation $\succ_{i}$ over $X$ is separable if for each $j \in N$ and each $x \in X$

${ }^{9}$ That is, $\succ_{i}$ is the strict part of a complete, transitive, and anti-symmetric relation over $X$.

This article is protected by copyright. All rights reserved. 
with $x_{j}=0, x+e^{j} \succ_{i} x$ if and only if $e^{j} \succ_{i} 0_{1 \times n}$. Let $\mathcal{S}$ be the set of all separable strict preference relations over $X$. Given preference relations $\succ_{i}, \succ_{i}^{\prime}$, and $\succ_{i}^{\prime \prime}$ over $X$, say that $\succ_{i}^{\prime \prime}$ is between $\succ_{i}$ and $\succ_{i}^{\prime}$ if for all distinct $x, y \in X, x \succ_{i} y$ and $x \succ_{i}^{\prime} y$ imply $x \succ_{i}^{\prime \prime} y \cdot{ }^{10}$ In words, whenever $\succ_{i}$ and $\succ_{i}^{\prime}$ agree on the preferences over a pair of decisions, $\succ_{i}^{\prime \prime}$ has the same preferences over the pair. A binary relation $\succ_{i}$ over $X$ is often identified with its graph in $X^{2}: \boldsymbol{G}\left(\succ_{i}\right) \equiv\left\{(x, y) \in X^{2}: x \succ_{i} y\right\}$. Expressed in terms of graphs, $\succ_{i}^{\prime \prime}$ is between $\succ_{i}$ and $\succ_{i}^{\prime}$ if and only if $G\left(\succ_{i}\right) \cap G\left(\succ_{i}^{\prime}\right) \subseteq G\left(\succ_{i}^{\prime \prime}\right)$. Given $p_{i} \in \mathcal{D}$, let $\mathcal{S}\left(\boldsymbol{p}_{\boldsymbol{i}}\right)$ be the set of all separable strict preference relations over $X$ that top-rank $p_{i}$. It $\bigcirc$ is simple to check that for each $p_{i} \in \mathcal{D}, G\left(P_{i}\right)=\bigcap_{\succ_{i} \in \mathcal{S}\left(p_{i}\right)} G\left(\succ_{i}\right)$. Therefore, $P_{i}$ consists of precisely those parts that lie between all separable strict preference relations over $X$ that top-rank $p_{i}$.

In essence, the preference extension captures the most information on preferences contained in opinions. Similar approaches are available in other literature. Given a preference relation over alterantives, Bossert and Sprumont (2014) derive preferences over preference relations using the betweenness notion. In the context of voting, Gibbard (1977) extends preferences over sure outcomes to preferences over lotteries defined on those outcomes using first-order stochastic dominance. Bogomolnaia and Moulin (2001) adopt the latter method to design ordinal mechanisms that allocate objects. Section 1 has a detailed discussion on the related literature.

\section{Incentives}

With preferences defined, we can now consider properties of rules that refer to these preferences. The first of these properties deals with incentive compatibility: the decision an agent gets by truthfully reporting his opinion should be weakly preferred to all the other decisions he gets by lying.

Strategy-proofness: For each $p \in \mathcal{D}^{N}$, each $i \in N$, and each $p_{i}^{\prime} \in \mathcal{D}$, either $\varphi(p)=$ $\varphi\left(p_{i}^{\prime}, p_{-i}\right)$ or $\varphi(p) P_{i} \varphi\left(p_{i}^{\prime}, p_{-i}\right)$.

One can formulate an alternative incentive property as follows: for each $p \in \mathcal{D}^{N}$ and each $i \in N$, there is no $p_{i}^{\prime} \in \mathcal{D}$ such that $\varphi\left(p_{i}^{\prime}, p_{-i}\right) P_{i} \varphi(p)$. Since the preference extension only gives an incomplete preference relation over $X$, this property is weaker than strategy-proofness. A rule may violate strategy-proofness just because $\varphi(p)$ and ${ }^{10}$ The original definition of betweenness by Grandmont (1978) allows for indifferences. The definition simplifies to ours for strict preferences.

This article is protected by copyright. All rights reserved. 
$\varphi\left(p_{i}^{\prime}, p_{-i}\right)$ are not comparable according to $P_{i}$ in some cases. Although the comparability requirement is demanding, it should be embedded in an incentive property to ensure immunity to manipulation by agents with any separable preferences (below we comment on the comparability requirement in detail when discussing implications of adjacent strategy-proofness). ${ }^{11}$ In this regard, strategy-proofness is a more appropriate notion than the above alternative property.

According to the definition of strategy-proofness, agents are free to report any opinion in the domain. We can formulate a similar but weaker property by restricting lies to be "close" to the truth. To define such property, we first make precise the meaning of closeness in $\mathcal{D}$. For each pair $p_{i}, p_{i}^{\prime} \in \mathcal{D}, p_{i}$ and $p_{i}^{\prime}$ are adjacent if there is exactly one $j \in N$ such that $p_{i j} \neq p_{i j}^{\prime}$. Opinions that are adjacent to the truth are the smallest lies, and we require that agents should not benefit from reporting those lies.

Adjacent strategy-proofness: For each $p \in \mathcal{D}^{N}$, each $i \in N$, and each $p_{i}^{\prime} \in \mathcal{D}$ such that $p_{i}$ and $p_{i}^{\prime}$ are adjacent, either $\varphi(p)=\varphi\left(p_{i}^{\prime}, p_{-i}\right)$ or $\varphi(p) P_{i} \varphi\left(p_{i}^{\prime}, p_{-i}\right)$.

While strategy-proofness has long been studied in the mechanism design literature, adjacent strategy-proofness is a relatively new concept (Carroll, 2012; Sato, 2013). In the standard social choice model where agents report strict preferences over alternatives, these properties are equivalent, regardless of whether the social choice rule is deterministic (Sato, 2013) or probabilistic (Carroll, 2012; Cho, 2016). Our first result shows that a similar equivalence extends to the group identification model.

Proposition 1 A rule is strategy-proof if, and only if, it is adjacent strategy-proof.

Proof. For the proof, we need the following concepts. Let $p_{i}, p_{i}^{\prime} \in \mathcal{D}$. First, a path from $\boldsymbol{p}_{\boldsymbol{i}}$ to $\boldsymbol{p}_{\boldsymbol{i}}^{\prime}$ in $\mathcal{D}$ is a sequence of opinions $\left\{p_{i}^{0}, p_{i}^{1}, \ldots, p_{i}^{k}\right\}$ in $\mathcal{D}$ such that (i) $p_{i}^{0}=p_{i}$ and $p_{i}^{k}=p_{i}^{\prime}$; and (ii) for each $h \in\{0,1, \ldots, k-1\}, p_{i}^{h}$ and $p_{i}^{h+1}$ are adjacent. For the path $\left\{p_{i}^{0}, p_{i}^{1}, \ldots, p_{i}^{k}\right\}$ from $p_{i}$ to $p_{i}^{\prime}$ in $\mathcal{D}$, we call $k$ the length of the path. Let $\boldsymbol{d}\left(\boldsymbol{p}_{\boldsymbol{i}}, \boldsymbol{p}_{\boldsymbol{i}}^{\prime}\right)$ denote the length of the shortest path from $p_{i}$ to $p_{i}^{\prime}$ in $\mathcal{D}$.

Consider the following auxiliary axiom, according to which for each natural number $m \in \mathbb{N}$, no agent benefits from reporting an opinion whose distance from the truth according to $d(\cdot, \cdot)$ is at most $m$.

Within- $\boldsymbol{m}$ strategy-proofness: For each $p \in \mathcal{D}^{N}$, each $i \in N$, and each $p_{i}^{\prime} \in \mathcal{D}$ such that $d\left(p_{i}, p_{i}^{\prime}\right) \leq m$, either $\varphi(p)=\varphi\left(p_{i}^{\prime}, p_{-i}\right)$ or $\varphi(p) P_{i} \varphi\left(p_{i}^{\prime}, p_{-i}\right)$.

\footnotetext{
${ }^{11} \mathrm{~A}$ similar issue arises in the context of object assignment where agents compare lotteries based on incomplete preference relations that are obtained by first-order stochastic dominance (Bogomolnaia and Moulin, 2001).
}

This article is protected by copyright. All rights reserved. 
To prove Proposition 1, it suffices to show that for each $m \in \mathbb{N}$, within- $m$ strategyproofness implies within- $(m+1)$ strategy-proofness. Let $\varphi$ be a within- $m$ strategy-proof rule. To show that $\varphi$ is within- $(m+1)$ strategy-proof, let $p \in \mathcal{D}^{N}, i \in N$, and $p_{i}^{\prime} \in \mathcal{D}$ be such that $d\left(p_{i}, p_{i}^{\prime}\right)=m+1$. Consider a path $\left\{p_{i}^{0}, p_{i}^{1}, \cdots, p_{i}^{m}, p_{i}^{m+1}\right\}$ from $p_{i}$ to $p_{i}^{\prime}$ in $\mathcal{D}$ with the property that (i) $p_{i}^{0}=p_{i}$, (ii) $p_{i}^{m+1}=p_{i}^{\prime}$; and (iii) for each $h \in\{0,1, \ldots, m\}$, $p_{i}^{h}$ and $p_{i}^{h+1}$ differ exactly in one entry. This path exists because $\mathcal{D}=\{0,1\}^{N}$. For simplicity, let $x \equiv \varphi(p), x^{\prime} \equiv \varphi\left(p_{i}^{\prime}, p_{-i}\right)$, and $\hat{x} \equiv \varphi\left(\hat{p}_{i}, p_{-i}\right)$, where $\hat{p}_{i} \equiv p_{i}^{m}$.

If $\hat{x}=x^{\prime}$, then by within- $m$ strategy-proofness, either $x=\hat{x}=x^{\prime}$ or $x P_{i} \hat{x}=x^{\prime}$. Thus, assume that $\hat{x} \neq x^{\prime}$. Because $\hat{p}_{i}$ and $p_{i}^{\prime}$ are adjacent, there is exactly one $j \in N$ such that $\hat{p}_{i j} \neq p_{i j}^{\prime}$. First, we show that for each $k \in N \backslash\{j\}, \hat{x}_{k}=x_{k}^{\prime}$. Suppose, by contradiction, that for some $k \in N \backslash\{j\}, \hat{x}_{k} \neq x_{k}^{\prime}$. Note that $\hat{p}_{i k}=p_{i k}^{\prime}$. First, suppose that $\hat{x}_{k}=\hat{p}_{i k}=p_{i k}^{\prime} \neq x_{k}^{\prime}$. By adjacent strategy-proofness, $x^{\prime} P_{i}^{\prime} \hat{x}$. By the definition of the preference extension, $p_{i k}^{\prime} \neq x_{k}^{\prime}$ implies $p_{i k}^{\prime} \neq \hat{x}_{k}$, a contradiction. Second, suppose that $\hat{x}_{k} \neq \hat{p}_{i k}=p_{i k}^{\prime}=x_{k}^{\prime}$. By adjacent strategy-proofness, $\hat{x} \hat{P}_{i} x^{\prime}$. By the definition of the preference extension, $\hat{p}_{i k} \neq \hat{x}_{k}$ implies $\hat{p}_{i k} \neq x_{k}^{\prime}$, a contradiction.

Second, we prove that $\hat{p}_{i j}=\hat{x}_{j}$. Suppose not. By adjacent strategy-proofness, $\hat{x} \hat{P}_{i} x^{\prime}$. By the definition of the preference extension, $\hat{p}_{i j} \neq \hat{x}_{j}$ implies $\hat{p}_{i j} \neq x_{j}^{\prime}$. Thus, $\hat{x}_{j}=$ $x_{j}^{\prime}$. Combining this with the result in the previous paragraph, we have that $\hat{x}=x^{\prime}$, contradicting the initial hypothesis that $\hat{x} \neq x^{\prime}$.

Finally, third, we show that $\hat{x} P_{i} x^{\prime}$. Recall that the path $\left\{p_{i}^{0}, \ldots, p_{i}^{m}, p_{i}^{m+1}\right\}$ from $p_{i}$ to $p_{i}^{\prime}$ in $\mathcal{D}$ is the shortest path from $p_{i}$ to $p_{i}^{\prime}$ in $\mathcal{D}$ and that any two successive opinions in the path differ exactly in one entry. Since $\hat{p}_{i j} \neq p_{i j}^{\prime}$, this implies that $p_{i j}=\hat{p}_{i j} \neq p_{i j}^{\prime}$. Combined with the arguments in the previous two paragraphs, it follows that (i) $p_{i j}=$ $\hat{p}_{i j}=\hat{x}_{j} \neq x_{j}^{\prime}$; and (ii) for each $k \in N \backslash\{j\}, \hat{x}_{k}=x_{k}^{\prime}$. Thus, $\hat{x} P_{i} x^{\prime}$.

Proposition 1 plays a key role in unveiling the structure of strategy-proof group identification rules. Indeed, consider a rule $\varphi$. Let $p \in \mathcal{D}^{N}$ and $i \in N$. Suppose that agent $i$ changes his opinion from $p_{i}$ to an adjacent opinion $p_{i}^{\prime} \in \mathcal{D}$ such that for some $j \in N, p_{i j} \neq p_{i j}^{\prime}$. Assume that $\varphi$ is adjacent strategy-proof. If $\varphi$ returns different decisions for $p$ and $\left(p_{i}^{\prime}, p_{-i}\right)$, then by adjacent strategy-proofness, the two decisions should differ only in agent $j$ 's membership. To see this, suppose that $\varphi(p) \neq \varphi\left(p_{i}^{\prime}, p_{-i}\right)$ and let $k \in N \backslash\{j\}$. Adjacent strategy-proofness implies that $\varphi(p) P_{i} \varphi\left(p_{i}^{\prime}, p_{-i}\right)$ and $\varphi\left(p_{i}^{\prime}, p_{-i}\right) P_{i}^{\prime} \varphi(p)$. By adjacency of $p_{i}$ and $p_{i}^{\prime}, p_{i k}=p_{i k}^{\prime}$. If $\varphi_{k}(p) \neq \varphi_{k}\left(p_{i}^{\prime}, p_{-i}\right)$, then either $p_{i k}=p_{i k}^{\prime}=\varphi_{k}(p) \neq \varphi_{k}\left(p_{i}^{\prime}, p_{-i}\right)$ or $\varphi_{k}(p) \neq \varphi_{k}\left(p_{i}^{\prime}, p_{-i}\right)=p_{i k}=p_{i k}^{\prime}$, so that either $\varphi(p) P_{i} \varphi\left(p_{i}^{\prime}, p_{-i}\right)$ or $\varphi\left(p_{i}^{\prime}, p_{-i}\right) P_{i}^{\prime} \varphi(p)$ is violated. ${ }^{12}$ Thus, $\varphi_{k}(p)=\varphi_{k}\left(p_{i}^{\prime}, p_{-i}\right)$. Now

${ }^{12}$ For a weaker incentive property that allows the case where $\varphi(p)$ and $\varphi\left(p_{i}^{\prime}, p_{-i}\right)$ are not comThis article is protected by copyright. All rights reserved. 
once we know that for each $k \in N \backslash\{j\}, \varphi_{k}(p)=\varphi_{k}\left(p_{i}^{\prime}, p_{-i}\right)$, it follows that the difference between decisions $\varphi(p)$ and $\varphi\left(p_{i}^{\prime}, p_{-i}\right)$ should be the same as the difference between opinions $p_{i}$ and $p_{i}^{\prime}$. Therefore, $\varphi_{j}(p)=p_{i j} \neq p_{i j}^{\prime}=\varphi_{j}\left(p_{i}^{\prime}, p_{-i}\right)$ and for each $k \in N \backslash\{j\}$, $\varphi_{k}(p)=\varphi_{k}\left(p_{i}^{\prime}, p_{-i}\right)$. It is simple to see that the latter behavior of $\varphi$ is not just necessary but also sufficient for adjacent strategy-proofness. Now by Proposition 1, it is also necessary and sufficient for strategy-proofness. Thus, we have the following characterization of strategy-proof rules.

Corollary 1 A rule $\varphi$ is strategy-proof if, and only if, for each $p \in \mathcal{D}^{N}$, each $i \in N$, and each $p_{i}^{\prime} \in \mathcal{D}$ such that $p_{i}$ and $p_{i}^{\prime}$ are adjacent, with $p_{i j} \neq p_{i j}^{\prime}$ for some $j \in N$, either (i) $\varphi(p)=\varphi\left(p_{i}^{\prime}, p_{-i}\right)$, or (ii) $\varphi_{j}(p)=p_{i j} \neq p_{i j}^{\prime}=\varphi_{j}\left(p_{i}^{\prime}, p_{-i}\right)$ and for each $k \in N \backslash\{j\}$, $\varphi_{k}(p)=\varphi_{k}\left(p_{i}^{\prime}, p_{-i}\right)$.

Corollary 1 relates strategy-proofness to two well-known properties in the literature on group identification (Samet and Schmeidler, 2003). The first property states that a rule should respond monotonically to changes in problems.

Monotonicity: For each pair $p, p^{\prime} \in \mathcal{D}^{N}$ such that $p \geq p^{\prime}, \varphi(p) \geq \varphi\left(p^{\prime}\right)$.

The second property requires independence of decisions across agents. That is, it demands that to determine agent $i$ 's membership, the rule consider only the opinions about agent $i$.

Independence: For each $i \in N$ and each pair $p, p^{\prime} \in \mathcal{D}^{N}$ with $p^{i}=\left(p^{\prime}\right)^{i}, \varphi_{i}(p)=\varphi_{i}\left(p^{\prime}\right)$. By Corollary 1, it is simple to see that strategy-proofness implies monotonicity and independence. Our next result shows that the converse is also true.

Proposition 2 A rule is strategy-proof if, and only if, it is monotonic and independent. $^{13}$

Proof. (Sufficiency) Let $\varphi$ be a monotonic and independent rule. By Proposition 1, it is enough to show that $\varphi$ is adjacent strategy-proof. Let $p \in \mathcal{D}^{N}, i \in N$, and $p_{i}^{\prime} \in \mathcal{D}$ such that $p_{i}$ and $p_{i}^{\prime}$ are adjacent. Let $x \equiv \varphi(p)$ and $x^{\prime} \equiv \varphi\left(p_{i}^{\prime}, p_{-i}\right)$. We may assume parable according to $P_{i}$ or $P_{i}^{\prime}$ (see the discussion following the definition of strategy-proofness), $\varphi_{k}(p) \neq \varphi_{k}\left(p_{i}^{\prime}, p_{-i}\right)$ does not necessarily lead to a contradiction. This indicates that the comparability requirement in (adjacent) strategy-proofness plays an important role in inducing the characterized behavior of strategy-proof rules in Corollary 1.

${ }^{13}$ Similar results are available in the preference aggregation literature, though under different assumptions on preferences; see Nehring and Puppe (2007) for the domain of generalized single-peaked preferences and $\mathrm{Ju}(2003)$ for the domain of separable preferences. In our model, preferences are separable but incomplete.

This article is protected by copyright. All rights reserved. 
that $x \neq x^{\prime}$ (otherwise, the proof is complete). Since $p_{i}$ and $p_{i}^{\prime}$ are adjacent, there is exactly one $j \in N$ such that $p_{i j} \neq p_{i j}^{\prime}$. By independence, for each $k \in N \backslash\{j\}, x_{k}=x_{k}^{\prime}$. If $p_{i j}<p_{i j}^{\prime}$, then by monotonicity and $x \neq x^{\prime}$, it follows that $x_{j}<x_{j}^{\prime}$. Thus, $x P_{i} x^{\prime}$. A similar argument applies to the case where $p_{i j}>p_{i j}^{\prime}$.

(Necessity) Let $\varphi$ be a strategy-proof rule. First, we show that $\varphi$ is monotonic. Let $p, p^{\prime} \in \mathcal{D}^{N}$ be such that $p \leq p^{\prime}$. By appealing to an induction argument, we may assume that there is exactly one pair $(i, j) \in N \times N$ such that $p_{i j} \neq p_{i j}^{\prime}$. Since $p \leq p^{\prime}$, $p_{i j}=0 \neq 1=p_{i j}^{\prime}$. Now by Corollary 1 , either (i) $\varphi(p)=\varphi\left(p^{\prime}\right)$; or (ii) $\varphi_{j}(p)=p_{i j}=0 \neq$ $1=p_{i j}^{\prime}=\varphi_{j}\left(p^{\prime}\right)$ and for each $k \in N \backslash\{j\}, \varphi_{k}(p)=\varphi_{k}\left(p^{\prime}\right)$. Thus, $\varphi(p) \leq \varphi\left(p^{\prime}\right)$.

Second, we show that $\varphi$ is independent. Let $p, p^{\prime} \in \mathcal{D}^{N}$ and $i \in N$ be such that $p^{i}=\left(p^{\prime}\right)^{i}$. By appealing to an induction argument, we may assume that there is exactly one pair $(k, j) \in N \times(N \backslash\{i\})$ such that $p_{k j} \neq p_{k j}^{\prime}$. Then by Corollary 1 , for each $\ell \in N \backslash\{j\}$, $\varphi_{\ell}(p)=\varphi_{\ell}\left(p^{\prime}\right)$. In particular, $\varphi_{i}(p)=\varphi_{i}\left(p^{\prime}\right)$.

Çengelci and Sanver (2010) characterize the family of rules satisfying monotonicity and independence. ${ }^{14}$ By Proposition 2, this family is also the family of strategy-proof rules. To describe these rules, we borrow the following concepts from Barberà et al. (1991). A coalition is a subset of the set of agents $N$ (which is allowed to be empty or $N$ itself). A committee for agent $i$ is a collection $\mathcal{W}_{i}$ of coalitions satisfying the following condition: for each pair $M, M^{\prime} \subseteq N$, if $M \in \mathcal{W}_{i}$ and $M \subseteq M^{\prime}$, then $M^{\prime} \in \mathcal{W}_{i}$. Elements of $\mathcal{W}_{i}$ are called winning coalitions with respect to $\mathcal{W}_{i}$.

Let $\mathcal{W} \equiv\left(\mathcal{W}_{i}\right)_{i \in N}$ be a profile of committees. The voting-by-committees rule with respect to $\mathcal{W}$, denoted $\varphi^{\mathcal{W}}$, is defined as follows: for each $p \in \mathcal{D}^{N}$ and each $i \in N$, $\varphi_{i}^{\mathcal{W}}(p)=1$ if, and only if, $N_{1}(p, i) \in \mathcal{W}_{i}$. In words, a voting-by-committees rule is one that qualifies each agent $i$ if, and only if, the agents who consider agent $i$ to be a member of the group form a winning coalition with respect to $\mathcal{W}_{i} \cdot{ }^{15}$ Now combining Proposition 2 with Çengelci and Sanver (2010), it follows that the voting-by-committees rules are the only strategy-proof rules in our framework.

Theorem 1 A rule is strategy-proof if, and only if, it is a voting-by-committees rule.

It is worth pointing out that Theorem 1 can easily be extended to the setup of Barberà et al. (1991), where alternatives are abstract objects instead of individuals. Rename the

${ }^{14}$ The notion of monotonicity in Çengelci and Sanver (2010) is slightly weaker than our notion of monotonicity. However, in the presence of independence, the two are equivalent.

${ }^{15}$ Notice that a committee is not required to be proper (or strong). Hence, the case in which each coalition is winning, i.e., $\mathcal{W}_{i}=2^{N}$ (and the case in which no coalition is winning, i.e., $\mathcal{W}_{i}=\emptyset$, respectively), is allowed. As a result, a voting-by-committees rule can be degenerate for an agent, in the sense that for all problems, the rule returns the same decision for him.

This article is protected by copyright. All rights reserved. 
incentive property in Barberà et al. (1991) to BSZ-strategy-proofness. The paper shows that when agents have complete and separable preferences, the family of voting-bycommittees rules is characterized by BSZ-strategy-proofness and a full-range condition known as voter sovereignty. ${ }^{16}$ In contrast, Theorem 1 indicates that with our preference extension (that orders decisions partially), the same family of rules is characterized by a stronger notion of strategy-proofness alone, without voter sovereignty.

\section{Fairness}

As pointed out in the Introduction, we are not only interested in incentive compatible mechanisms for group identification but also in mechanisms that treat the agents fairly. In this section, we define two properties of fairness. The first property concerns how a rule should treat "equal" agents. Given a problem $p \in \mathcal{D}^{N}$, we say that two arbitrary agents $i$ and $j$ are equal if they have the same opinion (i.e., $p_{i}=p_{j}$ ) and all agents have the same opinion about them (i.e., $\left.p^{i}=p^{j}\right) \cdot{ }^{17}$ A reasonable request is that the decisions for two equal agents should coincide.

Equal treatment of equals: For each pair $i, j \in N$ and each $p \in \mathcal{D}^{N}$ such that $p_{i}=p_{j}$ and $p^{i}=p^{j}, \varphi_{i}(p)=\varphi_{j}(p)$.

We seek to identify the family of rules satisfying strategy-proofness and equal treatment of equals. By Theorem 1, these rules are the voting-by-committees rules. To find the properties that the committees $\mathcal{W}=\left(\mathcal{W}_{i}\right)_{i \in N}$ should have in order for the rule $\varphi^{\mathcal{W}}$ to satisfy equal treatment of equals, take any two agents $i, j \in N$. If $\mathcal{W}_{i}=\mathcal{W}_{j}$, then whenever agents $i$ and $j$ are equal, their membership decisions are the same. Thus, any potential violation of equal treatment of equals is due to the winning coalitions in $\mathcal{W}_{i} \backslash \mathcal{W}_{j}$ and $\mathcal{W}_{j} \backslash \mathcal{W}_{i}$

Without loss of generality, let $M \in \mathcal{W}_{i} \backslash \mathcal{W}_{j}$. If either $M \cap\{i, j\}=\emptyset$ or $\{i, j\}$, we can construct a problem $p \in \mathcal{D}^{N}$ such that agents $i$ and $j$ are equals in $p$ and $M$ is the set of agents who view agent $i$ (and $j$ ) as a member. Because $M \in \mathcal{W}_{i} \backslash \mathcal{W}_{j}$, for such $p, \varphi^{\mathcal{W}}$ decides that agent $i$ is a member and agent $j$ is not, violating equal treatment of equals. Therefore, equal treatment of equals requires that for each $M \in \mathcal{W}_{i} \backslash \mathcal{W}_{j}$, $|M \cap\{i, j\}|=1$. In fact, this condition is also sufficient for equal treatment of equals.

${ }^{16}$ Voter sovereignty requires that for each alternative, there exist a preference profile for which the rule chooses that alternative.

${ }^{17}$ These agents are called symmetric in Ksaher and Rubinstein (1997). Yet we reserve the term "symmetry" for the fairness concept introduced by Samet and Schmeidler (2003), which requires that the names of the agents should not affect decisions.

This article is protected by copyright. All rights reserved. 
Now say that a profile of committees $\mathcal{W} \equiv\left(\mathcal{W}_{i}\right)_{i \in N}$ is equitable if for each pair $i, j \in N$ and each set $M \in \mathcal{W}_{i} \backslash \mathcal{W}_{j},|M \cap\{i, j\}|=1$. A voting-by-equitable-committees rule is a voting-by-committees rule whose associated committees are equitable.

Theorem 2 A rule satisfies strategy-proofness and equal treatment of equals if, and only if, it is a voting-by-equitable-committees rule.

Proof. (Sufficiency) Let $\mathcal{W} \equiv\left(\mathcal{W}_{i}\right)_{i \in N}$ be a profile of equitable committees. By Theorem 1 , the voting-by-committees rule $\varphi^{\mathcal{W}}$ is strategy-proof. Now we show that $\varphi^{\mathcal{W}}$ satisfies equal treatment of equals. Let $p \in \mathcal{D}^{N}$ and $i, j \in N$ be such that $p_{i}=p_{j}$ and $p^{i}=p^{j}$. Let $M \equiv N_{1}(p, i)\left(=N_{1}(p, j)\right)$. Because $p_{i}=p_{j}$ and $p^{i}=p^{j}, p_{i i}=p_{i j}=p_{j j}=p_{j i}$. First, if $M \in \mathcal{W}_{i} \cap \mathcal{W}_{j}$, then $\varphi_{i}^{\mathcal{W}}(p)=\varphi_{j}^{\mathcal{W}}(p)=1$. Second, if $M \in\left(\mathcal{W}_{i} \cup \mathcal{W}_{j}\right)^{c}$, then $\varphi_{i}^{\mathcal{W}}(p)=\varphi_{j}^{\mathcal{W}}(p)=0$. Third, suppose, without loss of generality, that $M \in \mathcal{W}_{i} \backslash \mathcal{W}_{j}$. Since $p_{i i}=p_{i j}=p_{j j}=p_{j i}, M \cap\{i, j\}=\{i, j\}$ or $\emptyset$, a contradiction.

(Necessity) Let $\varphi$ be a rule satisfying strategy-proofness and equal treatment of equals. By Theorem 1 , there is a profile of committees $\mathcal{W} \equiv\left(\mathcal{W}_{i}\right)_{i \in N}$ such that $\varphi=\varphi^{\mathcal{W}}$. Now we show that $\mathcal{W}$ is equitable. Suppose, by contradiction, that there are $i, j \in N$ and $M \in \mathcal{W}_{i} \backslash \mathcal{W}_{j}$ such that $|M \cap\{i, j\}| \neq 1$. Then either $M \supseteq\{i, j\}$ or $M \cap\{i, j\}=\emptyset$. Let $p \in \mathcal{D}^{N}$ be such that $p_{i}=p_{j}, p^{i}=p^{j}$, and $N_{1}(p, i)=M$ (such $p$ can be constructed if and only if $|M \cap\{i, j\}| \neq 1)$. Since $M \in \mathcal{W}_{i} \backslash \mathcal{W}_{j}, \varphi_{i}(p)=\varphi_{i}^{\mathcal{W}}(p)=1$ and $\varphi_{j}(p)=$ $\varphi_{j}^{\mathcal{W}}(p)=0$, a contradiction.

As an alternative principle of fairness, we may request not only that equal agents be treated equally, but that the names of the agents never matter for group identification, regardless of whether they are equal or not. This is obviously stronger than equal treatment of equals, and it is formally expressed by renaming the set of agents, and then demanding that the social decisions before and after the renaming be the same. To be more precise, recall that a permutation of the set of agents is a one-to-one function $\pi: N \rightarrow N$. For each $p \in \mathcal{D}^{N}$, let $\boldsymbol{p}_{\boldsymbol{\pi}} \equiv\left(p_{\pi(i), \pi(j)}\right)_{i, j \in N}$ be the problem in the new names, and $\boldsymbol{\varphi}_{\boldsymbol{\pi}}(\boldsymbol{p}) \equiv\left(\varphi_{\pi(i)}(p)\right)_{i \in N}$ the decision in the new names.

Symmetry: For each $p \in \mathcal{D}^{N}$ and each permutation $\pi: N \rightarrow N, \varphi_{\pi}(p)=\varphi\left(p_{\pi}\right){ }^{18}$

By imposing symmetry, we obtain a subfamily of voting-by-equitable-committees rules, called the consent rules (Samet and Schmeidler, 2003). Let $s, t \in\{1, \ldots, n+1\}$ be such that $s+t \leq n+2$. The consent rule with $(s, t)$, denoted $\varphi^{s t}$, is defined as

${ }^{18}$ We borrow the term "symmetry" from Samet and Schmeidler (2003). The same term means equal treatment of equals in Kasher and Rubinstein (1997).

This article is protected by copyright. All rights reserved. 
follows. For each $p \in \mathcal{D}^{N}$ and each $i \in N$, (i) if $p_{i i}=1$, then $\left[\varphi_{i}^{s t}(p)=1\right.$ if, and only if, $\left.N_{1}(p, i) \geq s\right]$; and (ii) if $p_{i i}=0$, then $\left[\varphi_{i}^{s t}(p)=0\right.$ if, and only if, $\left.N_{0}(p, i) \geq t\right]$. The consent rules embody various degrees of liberalism and democracy. For instance, $s=t=1$ gives the liberal rule; $s=t=\left\lceil\frac{n+1}{2}\right\rceil$ the simple majority rule; and either $s=n$ or $t=n$ the rule with the unanimity requirement for socially approving selfqualification or self-disqualification, respectively. ${ }^{19}$ Samet and Schmeidler (2003) show that a rule is monotonic, independent, and symmetric if, and only if, it is a consent rule. $^{20}$ The following characterization of the consent rules directly follows from Samet and Schmeidler (2003) when monotonicity and independence are replaced by strategyproofness.

Theorem 3 A rule is strategy-proof and symmetric if, and only if, it is a consent rule.

Figure 1 illustrates the fact that the voting-by-equitable-committees rules are a superset of the consent rules and a subset of the voting-by-committees rules. Both inclusion relations are strict. For the former inclusion, let $n=3$ and consider the committees $\mathcal{W}_{1}=\mathcal{W}_{2}=\{\{1,2\}, N\}$ and $\mathcal{W}_{3}=\{N\}$. Then $\varphi^{\mathcal{W}}$ satisfies equal treatment of equals but not symmetry. For the latter inclusion, redefine the previous committees in such a way that $\mathcal{W}_{1}=\mathcal{W}_{2}=\{\{1,3\}, N\}$ and $\mathcal{W}_{3}=\{N\}$. This defines a voting-by-committees rule $\varphi^{\mathcal{W}}$ that satisfies strategy-proofness but not equal treatment of equals.

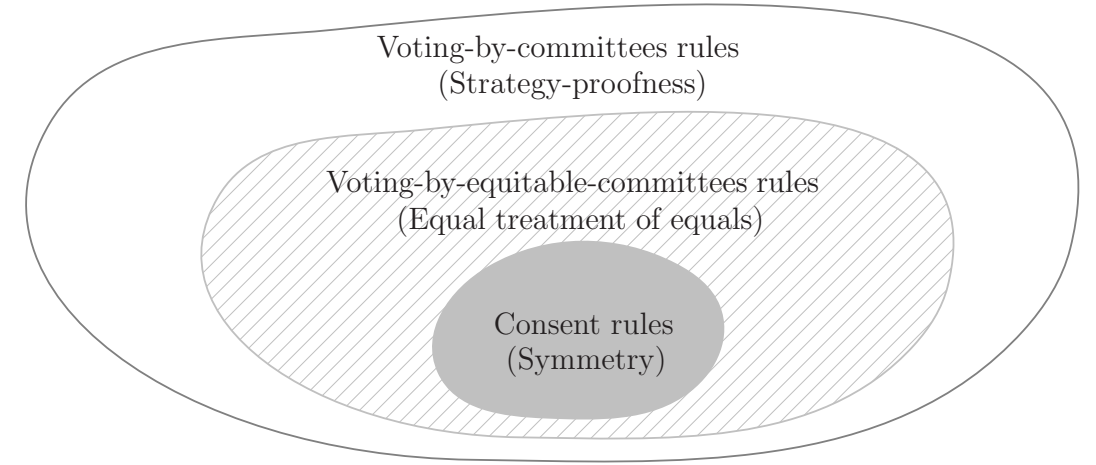

Figure 1: Main characterizations

${ }^{19}$ For each $x \in \mathbb{R},\lceil x\rceil$ denotes the smallest integer no less than $x$.

${ }^{20}$ Likewise, the voting-by-equitable-committees rules are characterized by monotonicity, independence, and equal treatment of equals, without need of referring to properties based on the preference extension.

This article is protected by copyright. All rights reserved. 


\section{$5 \quad$ Efficiency}

Let us finally define efficiency based on our preference extension and study its implications. Let $p \in \mathcal{D}^{N}$. For each pair $x, y \in X, \boldsymbol{x}$ Pareto dominates $\boldsymbol{y}$ for $\boldsymbol{p}$ if for each $i \in N, x P_{i} y .{ }^{21}$ For each $x \in X, x$ is efficient for $\boldsymbol{p}$ if there is no $y \in X$ such that $y$ Pareto dominates $x$ for $p$.

Efficiency: For each $p \in \mathcal{D}^{N}, \varphi(p)$ is efficient for $p$.

Recall that the preference relation $P_{i}$ recovered from the opinion $p_{i}$ is coarse. This means that our notion of Pareto dominance is strong and consequently, that our notion of efficiency is weak. ${ }^{22}$ However, when all separable preferences over $X$ are allowed, this is the strongest notion of efficiency that can be considered. ${ }^{23}$ Our next result shows that efficiency has an implication only for those agents about whom all agents have the same opinion. That is, a decision is efficient if, and only if, each agent whom all agents view as a member (and a non-member) is a member (and a non-member, respectively).

Proposition 3 Let $p \in \mathcal{D}^{N}$ and $x \in X$. Then $x$ is efficient for $p$ if, and only if, for each $i \in N$ such that $p_{1 i}=p_{2 i}=\ldots=p_{n i}, x_{i}=p_{1 i}$.

Proof. (Sufficiency) Let $p \in \mathcal{D}^{N}$ and $x \in X$. Assume that for each $i \in N$ such that $p_{1 i}=p_{2 i}=\ldots=p_{n i}, x_{i}=p_{1 i}$. Suppose, by contradiction, that there is $y \in X$ such that $y$ Pareto dominate $x$ for $p$. Let $N^{*} \equiv\left\{i \in N: p_{1 i}=p_{2 i}=\ldots=p_{n i}\right\}$. Clearly, $N^{*} \neq N$, and since $y \neq x$, there is $i \in N \backslash N^{*}$ such that $y_{i} \neq x_{i}$. Since $i \in N \backslash N^{*}$, there is $j \in N$ such that $p_{j i}=x_{i}$. Then this contradicts that $y P_{j} x$.

(Necessity) Let $p \in \mathcal{D}^{N}$ and $x \in X$. Assume that $x$ is efficient for $p$. Suppose, by contradiction, that for some $i \in N, p_{1 i}=p_{2 i}=\ldots=p_{n i}$ and $x_{i} \neq p_{1 i}$. Let $y \in X$ be such that $y_{i}=p_{1 i}$ and $y_{-i}=x_{-i}$. Then $y$ Pareto dominates $x$ for $p$, a contradiction.

Kasher and Rubinstein (1997) study implications of a property called "consensus". Formally, a rule $\varphi$ satisfies consensus if for each $i \in N$ and each $p \in \mathcal{D}^{N}$, (i) $p^{i}=1_{n \times 1}$

${ }^{21}$ The usual definition of Pareto dominance requires that (i) for each $i \in N$, either $x P_{i} y$ or $x=y$; and (ii) for some $j \in N, x P_{j} y$. Clearly, (i) and (ii) are equivalent to our definition of Pareto dominance.

${ }^{22}$ We can formulate another notion of efficiency for the setup where agents report preferences over $X$. This alternative notion is stronger than our definition. To see this, let $N=\{1,2\}$ and $p=((1,0),(0,1))$. Consider two decisions $(0,0)$ and $(1,1)$. According to our definition, $(0,0)$ is efficient. However, if each agent prefers $(1,1)$ to $(0,0),(1,1)$ Pareto dominates $(0,0)$.

${ }^{23}$ A similar comment applies to ordinal efficiency (Bogomolnaia and Moulin, 2001), a notion based on (partial) preferences over lotteries that are obtained from preferences over sure outcomes by applying first-order stochastic dominance.

This article is protected by copyright. All rights reserved. 
implies $\varphi_{i}(p)=1$; and (ii) $p^{i}=0_{n \times 1}$ implies $\varphi_{i}(p)=0$. Proposition 3 shows that efficiency is equivalent to consensus, and it offers a preference foundation for the latter. However, in the presence of strategy-proofness, efficiency reduces to a property weaker than consensus, called non-degeneracy, which demands, roughly speaking, that for no agent should the rule return the same decision for all problems.

Non-degeneracy: For each $i \in N$, there are $p, p^{\prime} \in \mathcal{D}^{N}$ such that $\varphi_{i}(p) \neq \varphi_{i}\left(p^{\prime}\right)$.

For strategy-proof rules, non-degeneracy implies efficiency. This is because strategyproofness implies monotonicity and independence, which, combined with nondegeneracy, pin down the decision for agent $i$ whenever $p_{1 i}=p_{2 i}=\ldots=p_{n i}$.

Proposition 4 A strategy-proof rule is efficient if, and only if, it is non-degenerate.

Finally, combining Theorem 2 and Proposition 4, we can characterize the family of rules that satisfies simultaneously the three properties of interest.

Theorem 4 A rule satisfies strategy-proofness, equal treatment of equals, and efficiency if, and only if, it is a non-degenerate voting-by-equitable-committees rule.

The proof of Theorem 4 follows easily from the fact that a voting-by-committees rule $\varphi^{\mathcal{W}}$ is non-degenerate if, and only if, for each $i \in N, \mathcal{W}_{i} \neq \emptyset$ and $\mathcal{W}_{i} \neq 2^{N}$.

\section{Discussion}

\subsection{A Weaker Incentive Property}

In Section 3, we introduced an incentive property where decisions are compared according to the preference extension of opinions. We use the preference extension there because we require that a rule should not be manipulated by agents with any separable preferences. With a smaller set of admissible preferences, one can formulate a weaker incentive property. As a simple case, we may assume that when comparing decisions, each agent takes a weighted sum of the differences between his opinion and decisions. We now explore the logical relation between strategy-proofness and the incentive property associated with this type of preferences.

Let $i \in N$. Let $\boldsymbol{w}_{\boldsymbol{i}} \equiv\left(w_{i j}\right)_{j \in N} \in \mathbb{R}_{++}^{N}$. For each pair $x, y \in\{0,1\}^{N}$, let $\|\boldsymbol{x}-\boldsymbol{y}\|_{\boldsymbol{w}_{\boldsymbol{i}}} \equiv$ $\sum_{j \in N} w_{i j} \cdot\left|x_{j}-y_{j}\right|$ be the weighted difference between $x$ and $y$. We assume that for each $i \in N$, each $p_{i} \in\{0,1\}^{N}$, and each pair $x, y \in\{0,1\}^{N}$, agent $i$ with opinion $p_{i}$

This article is protected by copyright. All rights reserved. 
weakly prefers $x$ to $y$ if and only if $\left\|p_{i}-x\right\|_{w_{i}} \leq\left\|p_{i}-y\right\|_{w_{i}}$. In contrast with the preference extension in Section 2, these preferences are complete (and separable). When $w_{i 1}=\cdots=w_{i n}$, agent $i$ 's preferences simply minimize the number of different entries in his opinion and a decision. ${ }^{24}$

Let $\boldsymbol{w} \equiv\left(w_{i}\right)_{i \in N}$. The following property requires that when each agent $i$ has the above preferences induced by $w_{i}$, no agent gain by lying.

$\boldsymbol{w}$-strategy-proofness: For each $p \in \mathcal{D}^{N}$, each $i \in N$, and each $p_{i}^{\prime} \in \mathcal{D},\left\|p_{i}-\varphi(p)\right\|_{w_{i}} \leq$ $\left\|p_{i}-\varphi\left(p_{i}^{\prime}, p_{-i}\right)\right\|_{w_{i}}$.

C2

It is clear that $w$-strategy-proofness is substantially weaker than strategy-proofness. When $w$-strategy-proofness is imposed, each agent $i$ is assumed to have very specific separable preferences induced by $w_{i}$ and $w$-strategy-proofness only requires that lying should not benefit any agent with such preferences. By contrast, strategy-proofness requires that lying should not benefit any agent with any separable preferences. However, with independence in place, the two incentive properties are equivalent.

Proposition 5 In the presence of independence, w-strategy-proofness is equivalent to strategy-proofness.

Proof. Let $\varphi$ be an independent rule. We only show that if $\varphi$ is $w$-strategy-proof, then it is strategy-proof. Assume that $\varphi$ is $w$-strategy-proof. By Proposition 2, it suffices to show that $\varphi$ is monotonic. Let $p, p^{\prime} \in \mathcal{D}^{N}$ be such that $p \leq p^{\prime}$. By appealing to an induction argument, we may assume that there is exactly one $(i, j) \in N \times N$ such that $p_{i j} \neq p_{i j}^{\prime}$. Let $x \equiv \varphi(p)$ and $x^{\prime} \equiv \varphi\left(p^{\prime}\right)$. Since $p \leq p^{\prime}, p_{i j}=0$ and $p_{i j}^{\prime}=1$. For each $k \in N \backslash\{j\}, p^{k}=\left(p^{\prime}\right)^{k}$, so that by independence, $x_{k}=x_{k}^{\prime}$. We may assume that $x_{j} \neq x_{j}^{\prime}$ (otherwise, the proof is complete). Now applying $w$-strategy-proofness to agent $i$ with true opinion $p_{i},\left\|p_{i}-x\right\|_{w_{i}} \leq\left\|p_{i}-x^{\prime}\right\|_{w_{i}}$. It is simple to check that if $x_{j}=1$ and $x_{j}^{\prime}=0$, the latter inequality is violated. Thus, $x_{j}=0$ and $x_{j}^{\prime}=1$, so that $x \leq x^{\prime}$.

Some corollaries follow from this proposition. By Theorem 1, the family of votingby-committees rules is characterized by $w$-strategy-proofness and independence. By Theorem 3, the consent rules are the only rules that satisfy $w$-strategy-proofness, independence, and symmetry.

${ }^{24}$ In that case, $\|\cdot\|_{w_{i}}$ is the Hamming metric.

This article is protected by copyright. All rights reserved. 


\subsection{Group Strategy-proofness}

In formulating strategy-proofness, we imagine a situation where an agent attempts to manipulate a rule unilaterally. Yet it is also conceivable for a group of agents to jointly misrepresent their private information to their benefit. By requiring that a rule be immune to all such joint misrepresentations, we obtain the following stronger incentive property. $^{25}$

Group strategy-proofness: For each $p \in \mathcal{D}^{N}$, each $M \subseteq N$, and each $p_{M}^{\prime} \in \mathcal{D}^{M}$, either (i) $\varphi(p)=\varphi\left(p_{M}^{\prime}, p_{N \backslash M}\right)$, or (ii) for each $i \in M, \varphi(p) P_{i} \varphi\left(p_{M}^{\prime}, p_{N \backslash M}\right){ }^{26}$

Pairwise strategy-proofness is a weakening of group strategy-proofness that restricts the set $M$ of deviating agents to contain at most two agents. ${ }^{27}$ Since the voting-bycommittees rules have been proved to be strategy-proof, one may wonder whether they are also pairwise or group strategy-proof. For a voting-by-committees rule, there is no condition that binds the relationship between the committee $\mathcal{W}_{i}$ for agent $i$ and the committee $\mathcal{W}_{j}$ for agent $j$. This opens up the possibility that the two agents get better off by jointly lying about their opinions. To see this point, consider the liberal rule. For simplicity, suppose that $N=\{1,2\}$. Choose $p \in \mathcal{P}^{N}$ with $p_{1}=(1,0)$ and $p_{2}=(0,1)$. Suppose that agents 1 and 2 deviate from their true opinions by reporting $p_{1}^{\prime}=(0,0)$ and $p_{2}^{\prime}=(0,0)$, respectively. Denote the liberal rule by $L$. Since $L(p)=(1,1)$ and $L\left(p_{1}^{\prime}, p_{2}^{\prime}\right)=(0,0)$, for each $i \in N$, the requirement $L(p) P_{i} L\left(p_{1}^{\prime}, p_{2}^{\prime}\right)$ is not satisfied. Thus, the liberal rule is not pairwise (or group) strategy-proof.

In fact, the observations in the above example of the liberal rule can be generalized. Given a committee $\mathcal{W}_{i}$ and a coalition $M \subseteq N$, say that $M$ is a minimal winning coalition (with respect to $\mathcal{W}_{i}$ ) if $M \in \mathcal{W}_{i}$ and for each $M^{\prime} \subsetneq M, M^{\prime} \notin \mathcal{W}_{i}$. Now consider a voting-by-committees rule $\varphi^{\mathcal{W}}$ with respect to $\mathcal{W}$. Suppose that $(*)$ there exist $G \equiv\left\{i_{1}, \cdots, i_{k}\right\} \subseteq N$ (with $k \geq 2$ ) and minimal winning coalitions $\left(M_{i}\right)_{i \in G}$ such that (i) for each $i \in G, M_{i} \in \mathcal{W}_{i}$; (ii) $G \subseteq \bigcap_{i \in G} M_{i}$; and (iii) the collection $\left\{M_{i} \backslash G: i \in G\right\}$ is pairwise disjoint. Let $p \in \mathcal{P}^{N}$ be such that

\footnotetext{
${ }^{25}$ Early contributions on group strategy-proofness include Green and Laffont (1979) and Dasgupta et al. (1979); see Moulin (1993) for a survey on the topic. As Barberà and Jackson (1995) note, group strategy-proofness may turn out to be equivalent to strategy-proofness in some models. Le Breton and Zaporozhets (2009) and Barberà et al. (2010) provide sufficient conditions for the equivalence.

${ }^{26}$ As was the case with strategy-proofness, our definition of group strategy-proofness requires comparability of the decisions obtained by telling the truth and lying.

${ }^{27}$ While pairwise strategy-proofness is weaker than group strategy-proofness by definition, its implications in some models may be just as strong as those of group strategy-proofness when combined with other axioms. E.g., Serizawa (2006) considers a version of pairwise strategy-proofness and strengthens existing characterizations in this fashion.
}

This article is protected by copyright. All rights reserved. 
(i) for each $h \in\{1, \cdots, k\}, p_{i_{h}}$ is the same as $1_{1 \times n}$ except $p_{i_{h}, i_{h-1}}=0$ (modulo $k$ );

(ii) for each $h \in\{1, \cdots, k\}$ and each $j \in M_{i_{h}} \backslash G, p_{j}$ is the same as $0_{1 \times n}$ except $p_{j, i_{h}}=1$; and

(iii) for each $j \in N \backslash\left(M_{i_{1}} \cup \cdots \cup M_{i_{h}}\right), p_{j}=0_{1 \times n}{ }^{28}$

For each $h \in\{1, \cdots, k\}$, since $\left\{j \in N: p_{j, i_{h}}=1\right\}=M_{i_{h}} \backslash\left\{i_{h+1}\right\} \notin \mathcal{W}_{i_{h}}$ (modulo $k$ ), $\varphi_{i_{h}}^{\mathcal{W}}(p)=0$. Now suppose that the agents in $G$ jointly misrepresent their opinions, by reporting $p_{i_{1}}^{\prime}=\cdots=p_{i_{k}}^{\prime}=1_{1 \times n}$. For the new problem $\left(p_{G}^{\prime}, p_{N \backslash G}\right)$, for each $i \in G$, $\varphi_{i}^{\mathcal{W}}\left(p_{G}^{\prime}, p_{N \backslash G}\right)=1$ and for each $j \in N \backslash G, \varphi_{j}^{\mathcal{W}}\left(p_{G}^{\prime}, p_{N \backslash G}\right)=\varphi_{j}^{\mathcal{W}}(p)$. Thus, for each $i \in G$, the requirement $\varphi^{\mathcal{W}}(p) P_{i} \varphi^{\mathcal{W}}\left(p_{G}^{\prime}, p_{N \backslash G}\right)$ of group strategy-proofness is not met. The latter is a violation of pairwise strategy-proofness if, furthermore, the set $G$ of deviating agents has two agents.

It is not difficult to construct a profile of committees $\mathcal{W}$ satisfying condition (*) above. The non-degenerate consent rules by Samet and Schmeidler (2003) are examples. In fact, we can show that each non-degenerate consent rule violates pairwise strategy-proofness. Consider a consent rule rule $\varphi^{s t}$ with quotas $(s, t)(s$ and $t$ satisfy $s+t \leq n+2)$. If $s \leq \frac{n+2}{2}$, then there is $G \subseteq N$ with $|G|=2$ and the desired conditions, so that $\varphi^{s t}$ is not pairwise strategy-proof. If $s>\frac{n+2}{2}$, then we can use the notion of duality, which is defined in the next subsection, to show that $\varphi^{s t}$ is not pairwise strategy-proof. ${ }^{29}$

\subsection{Duality}

In our setting, opinions and decisions about a given group are binary: each agent is either a member or a non-member of the group. We may exploit this partitioning property in identifying the group. For each $p_{i} \in \mathcal{D}$, let $\overline{\boldsymbol{p}}_{\boldsymbol{i}} \equiv 1_{1 \times n}-p_{i}$. Since $p_{i}$ is an opinion on who are members of the group, $\bar{p}_{i}$ is an opinion on who are non-members of the group. Similarly, for each $p \in \mathcal{D}^{N}$, let $\overline{\boldsymbol{p}} \equiv\left(\bar{p}_{i}\right)_{i \in N}$; and for each $x \in X$, let $\overline{\boldsymbol{x}} \equiv 1_{1 \times n}-x$. Suppose that the group we seek to identify is $A$. By the partitioning property, identifying members of $A$ is equivalent to identifying non-members of $A$. Thus, given a rule $\varphi$ and a problem $p$, we may apply $\varphi$ to $\bar{p}$ and take the complement of the decision $\varphi(\bar{p})$ to identify $A$. In effect, this is a way of constructing another rule from $\varphi$. Formally, the dual of a rule $\varphi$, denoted $\varphi^{d}$, is defined as follows: for each $p \in \mathcal{D}^{N}, \varphi^{d}(p)=\overline{\varphi(\bar{p})}$.

${ }^{28}$ In other words, $p$ is a problem at which for each $h \in\{1, \cdots, k\}$, agent $i_{h}$ is pivotal for agent $i_{h-1}$ 's membership (modulo $k$ ). Also, while we fully specify $p$ for notational convenience, a similar argument applies to any $\hat{p} \in \mathcal{P}^{N}$ such that for each $i \in G, \hat{p}^{i}=p^{i}$.

${ }^{29}$ Suppose that $s>\frac{n+2}{2}$. Since $t \leq \frac{n+2}{2}$, a similar argument implies that $\varphi^{t s}$ is not pairwise strategyproof. But $\varphi^{t s}$ is the dual of $\varphi^{s t}$ (Samet and Schmeidler, 2003) and a rule is pairwise (group) strategyproof if and only if its dual is. Thus, $\varphi^{s t}$ is not pairwise strategy-proof.

This article is protected by copyright. All rights reserved. 
We also say that $\boldsymbol{\varphi}$ and $\varphi^{d}$ are dual. In general, a rule may differ from its dual. The following axiom due to Samet and Schmeidler (2003) requires that the two coincide. ${ }^{30}$

Self-duality: For each $p \in \mathcal{D}^{N}, \varphi(p)=\overline{\varphi(\bar{p})}$.

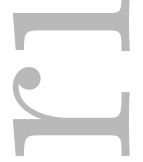

In essence, self-duality says that if some systematic method (rule) is used to identify a group under question, the same method should be used to identify the complement of the group. While the motivation for self-duality is solid, the property brings along rather unexpected conditions on rules. This is most clearly seen when we connect the preference restriction of separability with strategy-proofness. Under the separability restriction, agent $i$ 's opinion $p_{i}$ is interpreted as his most preferred decision in $X$. Then what decision should a strategy-proof rule choose when agent $i$ submits an opinion $\bar{p}_{i}$ that induces $\bar{P}_{i}$, the inverse of his true preferences $P_{i}$ ? With self-duality in place, the chosen decision should be the least preferred among all the decisions agent $i$ can get by manipulating. That is, $(*)$ for each $p \in \mathcal{D}^{N}$, each $i \in N$, and each $p_{i}^{\prime} \in \mathcal{D}$, either $\varphi\left(p_{i}^{\prime}, p_{-i}\right)=\varphi\left(\bar{p}_{i}, p_{-i}\right)$ or $\varphi\left(p_{i}^{\prime}, p_{-i}\right) P_{i} \varphi\left(\bar{p}_{i}, p_{-i}\right)$. Together with self-duality, strategyproofness implies (indeed, is equivalent to) condition $(*) .{ }^{31}$

The notion of duality reveals a simple, intuitive structure in the family of voting-bycommittees rules. Given a committee $\mathcal{W}_{i}$, consider a collection $\mathcal{W}_{i}^{d} \equiv\{N \backslash M: \forall M \subseteq$ $\left.N, M \notin \mathcal{W}_{i}\right\}$. Clearly, $\mathcal{W}_{i}^{d}$ satisfies the definition of committee and therefore let us call $\mathcal{W}_{i}^{d}$ the dual committee of $\mathcal{W}_{\boldsymbol{i}}$. Given a profile of committees $\mathcal{W} \equiv\left(\mathcal{W}_{i}\right)_{i \in N}$, let $\mathcal{W}^{d} \equiv\left(\mathcal{W}_{i}^{d}\right)_{i \in N}$. It is simple to check that for each profile of committees $\mathcal{W}, \varphi^{\mathcal{W}}$ and $\varphi^{\mathcal{W}^{d}}$ are dual; i.e., $\left(\varphi^{\mathcal{W}}\right)^{d}=\varphi^{\mathcal{W}^{d}}{ }^{32}$ The condition $\left(\varphi^{\mathcal{W}}\right)^{d}=\varphi^{\mathcal{W}^{d}}$ can be used to obtain the following characterization of the self-dual voting-by-committees rules (Çengelci and Sanver, 2010): for each profile of committees $\mathcal{W}, \varphi^{\mathcal{W}}$ is self-dual if, and only if, for each $i \in N$ and each $M \subseteq N$, exactly one of $M \in \mathcal{W}_{i}$ and $N \backslash M \in \mathcal{W}_{i}$ holds. $^{33}$

${ }^{30}$ In a different setup where the group under question enters the input for a rule, Miller (2008) considers a similar axiom called negation. While self-duality and negation can be motivated by the same reasoning about membership and non-membership for a group, their logical implications differ because of changes in the model.

${ }^{31}$ To prove this, recall that strategy-proofness can also be stated as follows: $(* *)$ for each $p \in \mathcal{D}^{N}$, each $i \in N$, and each $p_{i}^{\prime} \in \mathcal{D}$, either $\varphi\left(p_{i}, p_{-i}\right)=\varphi\left(\overline{p_{i}^{\prime}}, p_{-i}\right)$ or $\varphi\left(p_{i}, p_{-i}\right) P_{i} \varphi\left(\overline{p_{i}^{\prime}}, p_{-i}\right)$. Observe that $\varphi\left(p_{i}, p_{-i}\right) P_{i} \varphi\left(\overline{p_{i}^{\prime}}, p_{-i}\right)$ is equivalent to $\overline{\varphi\left(\overline{p_{i}^{\prime}}, p_{-i}\right)} P_{i} \overline{\varphi\left(p_{i}, p_{-i}\right)}$ and that by self-duality, $\overline{\varphi\left(\overline{p_{i}^{\prime}}, p_{-i}\right)}=$ $\varphi\left(p_{i}^{\prime}, \bar{p}_{-i}\right)$ and $\overline{\varphi\left(p_{i}, p_{-i}\right)}=\varphi\left(\bar{p}_{i}, \bar{p}_{-i}\right)$. Thus, condition $(* *)$ is equivalent to the following: for each $p \in \mathcal{D}^{N}$, each $i \in N$, and each $p_{i}^{\prime} \in \mathcal{D}, \varphi\left(p_{i}^{\prime}, \bar{p}_{-i}\right)=\varphi\left(\bar{p}_{i}, \bar{p}_{-i}\right)$ or $\varphi\left(p_{i}^{\prime}, \bar{p}_{-i}\right) P_{i} \varphi\left(\bar{p}_{i}, \bar{p}_{-i}\right)$. Now the latter condition is equivalent to condition $(*)$.

${ }^{32}$ To see this, let $p \in \mathcal{D}^{N}$ and $i \in N$. It suffices to show that $\varphi_{i}^{\mathcal{W}}(p)=0$ if and only if $\varphi_{i}^{\mathcal{V}^{d}}(\bar{p})=1$. Now note the equivalence of the following statements: $\varphi_{i}^{\mathcal{W}}(p)=0 \Longleftrightarrow N_{1}(p, i) \notin \mathcal{W}_{i} \Longleftrightarrow N_{0}(p, i) \in$ $\mathcal{W}_{i}^{d} \Longleftrightarrow N_{1}(\bar{p}, i) \in \mathcal{W}_{i}^{d} \Longleftrightarrow \varphi_{i}^{\mathcal{W}^{d}}(\bar{p})=1$.

${ }^{33}$ Çengelci and Sanver (2010) decompose self-duality into two conditions. Positive self-duality requires

This article is protected by copyright. All rights reserved. 


\section{References}

Arrow, K.J. (1951). Social Choice and Individual Values, Wiley, New York.

Athanasoglou, S. (2016). Strategyproof and efficient preference aggregation with Kemeny-based criteria. Games and Economic Behavior, 95, 156-167.

Barberà, S., Berga, D., Moreno, B. (2010), Individual versus group strategy-proofness: When do they coincide? Journal of Economic Theory 145, 1648-1674.

Barberà, S., Jackson, M. (1995). Strategy-proof exchange. Econometrica 63, 51-87.

Barberà, S., Sonnenschein, H., Zhou, L. (1991). Voting by committees. Econometrica 59, 595-609.

Bogomolnaia, A., Moulin, H. (2001). A new solution to the random assignment problems. Journal of Economic Theory 100, 295-328.

Bossert, W., Storcken, T. (1992). Strategy-proofness of social welfare functions: the use of the Kemeny distance between preference orderings, Social Choice and Welfare, 9, 345-360.

Bossert, W., Sprumont, Y. (2014). Strategy-proof preference aggregation: possibilities and characterizations, Games and Economic Behavior, 85, 109-126.

Carroll, G. (2012). When are local incentive constraints sufficient? Econometrica 80, 661-686.

Çengelci, M., Sanver, R. (2010). Simple collective identity functions. Theory and Decision $68,417-443$.

Cho, W.J. (2016). Incentive properties for ordinal mechanisms. Games and Economic Behavior 95, 168-177.

Cho, W.J., Ju, B.-G. (2015). Identifying groups in a Boolean algebra. Discussion Paper, Seoul National University. Available at: http://distributivejustice.snu.ac.kr/DP/2015/DP201516.pdf.

that for each $p \in \mathcal{P}$ and each $i \in N, \varphi_{i}(p)=1$ implies $\varphi_{i}(\bar{p})=0$. Negative self-duality requires that for each $p \in \mathcal{P}$ and each $i \in N, \varphi_{i}(p)=0$ implies $\varphi_{i}(\bar{p})=1$. The notion of self-duality presented here, due to Samet and Schmeidler (2003), is equivalent to the conjunction of positive and negative self-duality. Çengelci and Sanver (2010) study implications of positive and negative self-duality separately.

This article is protected by copyright. All rights reserved. 
Cho, W.J., Ju, B.-G. (2017). Multinary group identification. Theoretical Economics 12, 513-531.

Cho, W.J., Park, C.W. (2018). Fractional group identification. Journal of Mathematical Economics 77, 66-75.

Dasgupta, P., Hammond, P., Maskin, E. (1979). The implementation of social choice rules: Some general results on incentive compatibility. Review of Economic Studies $46,185-216$.

Dimitrov, D. (2011). The social choice approach to group identification, in: Consensual Processes, STUDFUZZ 267 (eds. E. Herrera-Viedma, J.L García-Lapresta, J. Kacprzyk, H. Nurmi, M. Fedrizzi, S. Zadrózny), Springer-Verlag, Berlin, 123-134.

Gibbard, A. (1977). Manipulation of schemes that mix voting with chance. Econometrica 45, 665-681.

Grandmont, J.-M. (1978). Intermediate preferences and the majority rule, Econometrica, 46, 317-330.

Green, J., Laffont, J.-J. (1979). On coalition incentive compatibility. Review of Economic Studies 46, 243-254.

Houy, N. (2007). "I want to be a J!": Liberalism in group identification problems. Mathematical Social Science 54, 59-70.

Ju, B.-G. (2003). A characterization of strategy-proof voting rules for separable weak orderings. Social Choice and Welfare 21, 469-499.

Ju, B.-G. (2005). Unanimity and resource monotonicity, Theory and Decision, 59, 1-17.

Ju, B.-G. (2013). On the characterization of liberalism by Samet and Schmeidler. Social Choice and Welfare 40, 359-366.

Kasher, A., Rubinstein, R. (1997). On the question "who is a j": A social choice approach. Logique Analyse 160, 385-395.

Kemeny, J.G. (1959). Mathematics without numbers, Daedalus 88, 575-591.

Kemeny, J.G., Snell, J.I. (1962). Mathematical Models in the Social Sciences, MIT Press, Cambridge.

This article is protected by copyright. All rights reserved. 
Le Breton, M., Zaporozhets, V. (2009). On the equivalence of coalitional and individual strategy-proofness properties. Social Choice and Welfare 33, 287-309.

List, C. (2008). Which worlds are possible? A judgment aggregation problem. Journal of Philosophical Logic, 37 (1), 57-65.

Miller, A.D. (2008). Group identification. Games and Economic Behavior 63, 188-202.

Moulin, H. (1993). On the fair and coalitions-strategyproof allocation of private goods. In: Binmore, K., Kirman, A., Tani, P. (eds), Frontiers of Game Theory, MIP Press, Cambridge.

Nehring, K., Puppe, C. (2007). The structure of strategy-proof social choice, Part I: General characterization and possibility results on median spaces. Journal of Economic Theory 135 (1), 269-305.

Samet, D., Schmeidler, D. (2003). Between liberalism and democracy. Journal of Economic Theory 110, 213-233.

Saporiti, A. (2012). A proof for 'who is a J' impossibility theorem. Economics Bulletin 32 (1), 494-501.

Sato, S. (2013). A sufficient condition for the equivalence of strategy-proofness and nonmanipulability by preferences adjacent to the sincere one. Journal of Economic Theory 148, 259-278.

Sato, S. (2015). Bounded response and the equivalence of nonmanipulability and independence of irrelevant alternatives. Social Choice and Welfare 44, 133-149.

Serizawa, S. (2006). Pairwise strategy-proofness and self-enforcing manipulation. Social Choice and Welfare 26, 305-331.

Sung, S.C., Dimitrov, D. (2005). On the axiomatic characterization of "who is a J?". Logique et Analyse 48, 101-112.

This article is protected by copyright. All rights reserved. 